\title{
Can self-pampering act as a buffer against depression in women? A cross-sectional study
}

\author{
Marianna Dalkou, ${ }^{1}$ Paraskevi Angelopoulou, ${ }^{1}$ Anthony Montgomery, ${ }^{2}$ \\ Efharis Panagopoulou' \\ ${ }^{1}$ Department of Medicine, Aristotle University of Thessaloniki; ${ }^{2}$ Department of Social and \\ Educational Policy, University of Macedonia, Greece
}

\begin{abstract}
Despite preliminary evidence that self-pampering can alleviate psychological burden that may lead to depression among women, no studies have so far examined the link between pampering and depression. The aim of this study was to explore the differential effect of pampering on depression depending on women's marital, parental, or caregiving status. A cross-sectional design was employed. The sample consisted of 154 women employees of the municipal authority of Thessaloniki, Greece. The Pampering Behaviors Inventory was developed for the purposes of the present study. Depression was assessed with the Hospital Anxiety and Depression Scale. Controlling for the effects of age, self-pampering was negatively related to depression $(p=.001)$. Married women, women with children, and women caregivers engaged in self-pampering activities less frequently. Married women who did not use pampering were more depressed than married women who used pampering $(\mathrm{p}=.002)$. Women with children who did not use pampering were more depressed than women with children who used pampering $(p=.004)$. Results of the present study contribute to a deeper understanding of the importance of self-pampering as a buffer against depression. Given the rising prevalence of depression today, it is essential to explore the potential of minimal interventions.
\end{abstract}

\footnotetext{
Correspondence: Angelopoulou Paraskevi, Department of Medicine, Aristotle University of Thessaloniki, 54124, Thessaloniki, Greece.

Tel.: +306947412690

E-mail: evi153@hotmail.com
}

Key words: women, pampering, depression, married, caregivers

Acknowledgments: The authors would like to thank all the women who voluntarily participated to this research

Contributions: The authors contributed equally.

Conflict of interests: The authors declare no potential conflict of interests.

Funding: none

Received for publication: 4 December 2018.

Accepted for publication: 27 November 2019.

This work is licensed under a Creative Commons AttributionNonCommercial 4.0 International License (CC BY-NC 4.0).

\section{Introduction}

The prevalence of depression is constantly rising. In Europe, the overall number of people with depression is 30 million and worldwide 350 million (Evans-Lacko \& Knapp, 2014). Depression has become the foremost underlying factor for disability with significant social and economic repercussions (EvansLacko \& Knapp, 2014). In terms of gender differences, it has been shown that the prevalence of depression is almost double in women than in men (Noble, 2005). Several risk factors have been identified increasing the possibility for depressive episodes in women, including divorce, job insecurity, lack of social support, and family history of depression (Evans-Lacko \& Knapp, 2014; Kasen, Cohen, Chen, \& Castille, 2003; Schoevers, Beekman, Deeg, Geerlings, Jonker, \& Van Tilburg, 2000). Additionally, women who take over the role of caregivers in their families are at higher risk of suffering from depression as a consequence of their chronic exposure to stressful events (Schulz \& Sherwood, 2008).

In terms of protective factors, being part of a relationship, can act as a buffer against depression mainly due to the emotional support provided (Plaisier et al., 2008). Similarly, it has been shown that problem sharing within a marriage has a beneficial effect on women's mental health (Wu \& Hart, 2002). Social and community networks can also act as a buffer against depression (Kuehner \& Buerger, 2005). Being a parent is also negatively associated with depression, depending on the available practical, financial, and social resources (Mirowsky \& Ross, 2002). For example, research has shown that single mothers have higher rates of depression compared to married mothers, a fact related to the greater exposure single mothers have to stressful life events, the lack of support and increased sense of vulnerability (Cairney, Boyle, Offord, \& Racine, 2003).

There is also evidence to suggest that taking care of one's appearance and self-pampering can alleviate the psychological burden that can lead to depression (Sharma \& Black, 2001). This is highlighted by the fact that places providing beauty services for women are recession-proof businesses (Straughan, 2010). For example, for the year 2013 the beauty and anti-aging industry was worth 1.02 billion dollars, and the spa industry 94 billon dollars (Global Wellness Institute, 2014). It is estimated that by 2017 the annual profit of the beauty salon industry will be roughly 50 billion dollars (Annual Report of the Beauty Salon Business Overview and Trends, 2012).

The rapid expansion of the beauty industry raises questions concerning the possible reasons behind women's visits to beauty salons. A qualitative study conducted in the US, revealed that women regularly visit beauty salons to share their problems and ask for personal advice (Anderson, Cimbal, \& Maile, 2009). The main topics of discussion concern either physical appearance, or 
personal relationships and employees claim that they can even detect symptoms of depression and dementia in their clients (Anderson, Cimbal, \& Maile, 2009; Linnan \& Ferguson, 2007). Indeed, health promotion interventions implemented in the above settings have been shown to be successful in enhancing screening behaviors concerning breast and cervical cancer (Linnan \& Ferguson, 2007). It is possible that the opportunity to devote time to one's self, or "escape" from daily routines, or the emotional support associated with the staff-customer relationship contribute towards an enhanced sense of emotional well-being, which can then influence health behaviors(Anderson, Cimbal, \& Maile, 2009; Straughan, 2010). Also, interviews with regular clients of health spas illustrate that after their visit, women report improved perceived health status and increased positive emotions (Little, 2013).

However, despite preliminary evidence proving that self-pampering can enhance psychological well-being, and the reported increasing usage of beauty salons and spa centers by women, no studies have so far examined the link between pampering and depression among women. However, in the same context, studies have examined the associations between cosmetic surgery and depression and have shown that cosmetic surgeries are chosen by women not only to improve their physical appearance but also to enhance their self-esteem and well-being (Honigman, Phillips, \& Castle, 2004). For example, a recent study showed that cosmetic therapies for glabella frown lines reduction, which use botulinum toxin (commonly known as Botox), seem to have a short-term (roughly 16 weeks) antidepressant effect on women (Young, 2013). It is therefore surprising that no studies have examined the effects of non-invasive self-pampering on women's mental health.

The aim of the present study was to examine the effect of selfpampering on depression among women. In specific, given existing literature on the role of marriage, parenthood and caregiving on depression, the study explored the differential effect of self-pampering on depression depending on women's marital, parental, or caregiving status. We hypothesized that self-pampering would be negatively related to depression. We also hypothesized that married women, women with children, and women caring for another member in their family would feel less depressed if they engaged in self-pampering activities. As self-pampering we defined taking care of one's appearance using non-invasive methods, at home or using external services. To control for the effects of working status only working women were included in the sample.

\section{Materials and Methods}

\section{Design}

A cross-sectional design was employed.

\section{Participants}

Participants consisted of all female employees working in a municipal administration office. Women currently on-leave, or under psychoactive medication were excluded from the study.

\section{Measures}

Demographic information included age, educational level, marital status, and number of children. Women were also asked to provide information in relation to if they were the main caregivers of a member of their family of friend, for the past 6 months.

The Pampering Behaviors Inventory (PBI) was developed for the purposes of the present study (see Appendix A1). The question- naire included 13 questions, assessing the extent to which women engage in several pampering behaviors at home, or using external services. Pampering activities at home, included putting make up on, doing their own hair and nails, while external pampering activities included visits to a hair salon, beauty salons, and spas. Questions were answered using a 7-point Likert scale ranging from 0 (being never) to 7 (being almost always). No items referring to minor or invasive cosmetic procedures were included. Similarly, in order to differentiate from the effects of exercise, no items were included referring to physical exercise or sport activities. The Cronbach's alpha for the PBI for the sample of this study was $\alpha=.82$.

The Hospital Anxiety and Depression Scale (HADS) by Zigmond,\& Snaith (1983) was used. The questionnaire includes 14 items assessing anxiety and depression. The questionnaire has been extensively used with different groups of general population to assess levels of anxiety and depression (Breeman, Cotton, Fielding, \& Jones, 2015).

\section{Procedure}

Ethical approval for the study was obtained from the Ethics Committee of the Aristotle University of Thessaloniki. The management board of the administration department was informed about the study and postal and electronic addresses of employees were requested. An email was then sent to all employees eligible for participation informing them about the study. No incentives were given for participation. Interested employees had to respond to the email confirming their consent to participate. Following that an interview was arranged where participants met with the same researcher and completed the questionnaire. All questionnaires were completed during working hours. Participants were informed that their participation was voluntary and that they were able to withdraw at any time. Finally, participants were informed about the confidentiality and anonymity of their data.

\section{Data analysis}

Pearson's correlation was used to assess the relationship between self-pampering and depression. In order to assess univariate differences in depression and pampering between the study's subgroups, independent samples t-tests were used. To assess the moderating role of pampering, the interaction term was calculated between marital status and pampering, parental status and pampering, and caregiving status and pampering. Three independent analyses of variance were conducted where the independent as well as the interaction effects were assessed (Field, 2009). To further identify differences between the study groups, in case the interaction effect was significant, four different subgroups were created for marriage, parental status, and caregiving status and self-pampering, using the median split for the self-pampering variable. Four independent analysis of variance were then conducted for each of the subgroups, using depression as an outcome variable. For all the statistical tests an alpha level of .05 was used and all $\mathrm{p}$ values represent two-tailed tests.

\section{Results}

Demographic information is shown in Table 1. The sample consisted of 154 women ( $65 \%$ response rate). $44.6 \%(\mathrm{~N}=69)$ of participants were aged between $25-34$ years old, $53.2 \%(\mathrm{~N}=82)$ of participants were married, while $48.1 \%(\mathrm{~N}=74)$ of them had children. Finally, $27.3 \%$ of participants $(\mathrm{N}=42)$ were caregivers of a 
family member or friend.

Results showed a negative correlation between self-pampering and depression $(\mathrm{r}=.-32, \mathrm{p}=.001)$. The relationship was still significant when controlling for the effect of age $(r=.-20, p=.001)$. Table 2 shows means and standard deviations, for depression and selfpampering for the different sample groups.

Independent samples t-test showed that married women had significantly higher levels of depression than non-married women [t $(152)=-2.956, p=.004]$. Women with children had significant higher scores of depression than women without children [ $\mathrm{t}$ (152) $=-3.559, \mathrm{p}=.001]$. Finally, results indicated that women caregivers had significantly higher levels of depression than women noncaregivers [t $(152)=-2.103, \mathrm{p}=.03]$.

Results also showed a significant difference in pampering between married and non-married women [ $\mathrm{t}(152)=2.153, \mathrm{p}=.03]$ with non-married women engaging in self-pampering activities more frequently than married women. Similarly, women without children engaged in self pampering activities more frequently than women with children $[\mathrm{t}(152)=2.207, \mathrm{p}=.02]$. Finally, women who were also caregivers engaged in less self-pampering activities than caregivers $[\mathrm{t}(152)=3.367, \mathrm{p}=.001]$.

The interaction effect of self-pampering and marital status on depression was significant $F(1,154)=3.774, p=.01$. The univariate analysis of variance for the different subgroups showed that the highest score on depression was reported by married women who engaged in no self-pampering activities, while the lowest score was reported by women who engaged in self-pampering activities. Also, married women who did not engage in self- pampering were more depressed than married women who engaged in self-pampering $(p=.002)$. Similarly, the interaction effect on depression between self-pampering and parental status was significant $[\mathrm{F}$ $(3,154)=6.350, p=.001]$. The highest score on depression was reported by women with children who engaged in no self-pampering activities, while the lowest score by women without children who engage in self-pampering behaviors $(p=.002)$. Also, women with children who did not use pampering were more depressed than women with children who used pampering $(\mathrm{p}=.004)$. No interaction effect was shown for pampering and caregiving status on depression (Table 3).

\section{Discussion}

The aim of this study was to examine the effect of self-pampering on depression among a sample of working women. The study also explored the moderating effects of self-pampering in the relationship between marital status, parental status, and caregiving status and depression. Results showed a negative association between self-pampering and depression, independent of age. Results also showed that married women had higher levels of depression and engaged in less self-pampering activities than nonmarried women. Women with children also had higher levels of depression and engaged in self-pampering less frequently than women without children. Women caregivers were more depressed and engaged in less self-pampering than women who were not caregivers. Finally, this study showed that married women, who engaged in self-pampering activities were less depressed than married women, who engaged in self-pampering activities less frequently. Similarly, women with children who engaged in self-pam-

Table 1. Demographic information ( $N=154)$.

\begin{tabular}{lc} 
Variables & Frequency (\%) \\
Education & $19(12.3)$ \\
Primary & $67(43.4)$ \\
Secondary & $68(44.1)$ \\
Tertiary & \\
Age $\quad$ & $69(44.8)$ \\
$25-34$ & $34(22.1)$ \\
$35-44$ & $28(18.2)$ \\
$45-54$ & $23(14.9)$ \\
55-64 & $72(46.8)$ \\
\hline Marital status & $82(53.2)$ \\
Non-married & \\
Married & $74(48.1)$ \\
Parental status & $80(51.9)$ \\
Children & \\
No children & $42(27.3)$ \\
\hline Caregiving status & $112(72.7)$ \\
Caregiver &
\end{tabular}

Table 2. Independent $t$-tests for the three groups for depression and pampering.

\begin{tabular}{|c|c|c|c|c|c|c|}
\hline Variables & Groups & M & SD & $\mathrm{t}$ & p & df \\
\hline \multirow[t]{6}{*}{ Depression } & Married $(n=82)$ & 11.73 & 2.98 & \multirow[t]{2}{*}{-2.956} & \multirow[t]{2}{*}{0.004} & \multirow[t]{2}{*}{152} \\
\hline & Unmarried $(n=72)$ & 10.29 & 3.04 & & & \\
\hline & Children $(\mathrm{n}=80)$ & 11.83 & 3.18 & \multirow[t]{2}{*}{-3.359} & \multirow[t]{2}{*}{0.001} & \multirow[t]{2}{*}{152} \\
\hline & No children $(n=74)$ & 10.21 & 2.76 & & & \\
\hline & Caregivers $(\mathrm{n}=42)$ & 11.90 & 2.98 & \multirow[t]{2}{*}{-2.103} & \multirow[t]{2}{*}{0.03} & \multirow[t]{2}{*}{152} \\
\hline & Non caregivers $(n=112)$ & 10.74 & 3.08 & & & \\
\hline \multirow[t]{6}{*}{ Pampering } & Married $(n=82)$ & 43.20 & 10.33 & \multirow[t]{2}{*}{2.153} & \multirow[t]{2}{*}{0.03} & \multirow[t]{2}{*}{152} \\
\hline & Unmarried $(\mathrm{n}=72)$ & 47.16 & 12.47 & & & \\
\hline & Children $(n=80)$ & 47.16 & 11.31 & \multirow[t]{2}{*}{2.207} & \multirow[t]{2}{*}{0.02} & \multirow[t]{2}{*}{152} \\
\hline & No children $(n=74)$ & 43.11 & 11.44 & & & \\
\hline & Caregivers $(n=42)$ & 40.11 & 11.08 & \multirow[t]{2}{*}{3.367} & \multirow[t]{2}{*}{0.001} & \multirow[t]{2}{*}{152} \\
\hline & Non caregivers $(n=112)$ & 46.91 & 11.17 & & & \\
\hline
\end{tabular}

$\mathrm{N}=154 . \mathrm{M}=$ Mean. $\mathrm{SD}=$ Standard Deviation. $p=$ significance value. $\mathrm{df}=$ degrees of Freedom. 
pering activities were less depressed than women with children who engaged in self-pampering less frequently.

Previous studies have shown contradictory findings concerning the relationship between marriage and depression. Our findings agree with studies showing that marriage can have an initial beneficial effect on women's physical and mental health, but subsequently due to increased responsibilities and very little personal time, women report a decline in physical health and an increase in depression levels (Keyes \& Goodman, 2006; Wu \& Hart, 2002). Results are also in agreement with studies showing that mothers report higher levels of depression as a result of worries about their children, and lack of time for themselves (Cairney, Boyle, Offord, \& Racine, 2003). Similarly, our results coincide with several studies showing that caregiving is related with worsened physical and mental health (Denno et al., 2013; Van Wijngaarden, Schene, \& Koeter, 2004). For example, in the study of Elliott and Shewchuk (2003) it was shown that women during the first year of caregiving of a family member or friend, have higher possibilities of experiencing depression especially if they lack the appropriate problemsolving abilities.

Our results show that overall self-pampering is associated with less depression among working women. It can also moderate the relationship between depression and marital and parental status. This could be attributed to the fact that self-pampering is a form of escaping from daily stressors. Previous studies have shown that women will seek for ways of escape as a coping mechanism to life stress and they acknowledge their need of uninterrupted time for self-care and constructive experiences (Keyes \& Goodman, 2006). Alternatively, the beneficial effects of pampering could be attributed to the fact that when women engage in self-pampering activities, they actually allow time for themselves. Previous studies have shown the importance of "self-time" as a buffer against distress especially for women (Sharma \& Black, 2001). These findings agree with preliminary evidence showing the impact of visits to beauty salons and spa's on women's emotional well-being (Honigman, Phillips, \& Castle, 2004).

Results of the study were based on a cross-sectional design using a non-randomised sample. As a result, no causal associations can be made concerning the reported relationships. Therefore, we cannot rule out the possibility that depressed women are less likely to utilize pampering. However, the study controlled for the possible confounding effects of age and working status. Future studies

Table 3. Means of depression among the different subgroup.

\begin{tabular}{lcc} 
Variables & M SD \\
Marital status & & \\
Non married-no self-pampering & 10.53 & 3.45 \\
Non married- self-pampering & 10.01 & 2.70 \\
Married- no self-pampering & 12.17 & 3.17 \\
Married- self-pampering & 11.18 & 2.68 \\
Parental status & & \\
No children- no self-pampering & 10.37 & 2.77 \\
No children- self-pampering & 10.02 & 2.77 \\
Children- no self-pampering & 12.65 & 3.37 \\
Children- self-pampering & 10.89 & 2.69 \\
\hline Caregiving status & & \\
Not caregiver- no self-pampering & 11.08 & 3.56 \\
Not caregiver- self-pampering & 10.47 & 2.65 \\
Caregiver- no self-pampering & 12.21 & 2.93 \\
Caregiver- self-pampering & 11.28 & 3.09 \\
\hline
\end{tabular}

$\mathrm{M}=$ Mean. $\mathrm{SD}=$ Standard Deviation. should examine further the relationship between self- pampering and depression using longitudinal designs. In addition, it would be interesting to explore the aforementioned relationships among men

\section{Implications for Practice and/or Policy}

Results of this study can be used to develop cost effective interventions aiming at promoting women's mental health. They highlight the fact that preventing strategies for psychological resilience could be an inherent part of everyday life. They also suggest the need for collaboration between different disciplines as well as sectors in terms of promoting women's mental health.

\section{Conclusions}

Results of the present study contribute to a deeper understanding of the importance of self-pampering as a buffer against depression. Given the rising prevalence of depression today, it is essential to explore protective mechanisms which can function preventively, especially among high risk groups. Given that self-pampering, either at home, or using external services is already a part of most women's lives, it also presents the added benefit of cost-effectiveness as a minimal intervention.

\section{References}

Anderson, K.A., Cimbal, A.M., \& Maile, J.J. (2009). Hairstylists' Relationships and Helping Behaviors With Older Adult Clients. Journal of Applied Gerontology, 29(3), 371-380.

Annual Report of the Beauty Salon Business Overview and Trends (2012). Retrieved from http://www.sbdcnet.org/smallbusiness-research-reports/beauty-salon-2012

Breeman, S., Cotton, S., Fielding, S., \& Jones, G.T. (2015). Normative data for the hospital anxiety and depression scale. Qual Life Res, 24(2), 391-398.

Cairney, J., Boyle, M., Offord, D. R., \& Racine, Y. (2003). Stress, social support and depression in single and married mothers. Social Psychiatry and Psychiatric Epidemiology, 38(8), 442449.

Denno, M.S., Gillard, P.J., Graham, G.D., DiBonaventura, M.D., Goren, A., Varon, S.F., \& Zorowitz, R. (2013). Anxiety and depression associated with caregiver burden in caregivers of stroke survivors with spasticity. Archives of Physical Medicine and Rehabilitation, 94(9), 1731-1736.

Elliott, T.R., \& Shewchuk, R.M. (2003). Social problem-solving abilities and distress among family members assuming a caregiving role. British Journal of Health Psychology, 8, 149-163.

Evans-Lacko, S., \& Knapp, M. (2014). Importance of social and cultural factors for attitudes disclosure and time off work for depression: Findings from a seven country European study on depression in the workplace: E91053. PLoS One, 9, 3.

Field, A.P. (2009). Discovering statistics using SPSS: And sex and drugs and rock ' $n$ ' roll. London: SAGE.

Global Wellness Institute (G.W.I.) (2014). Global spa and wellness economy monitor. Retrieved from http://www. globalspaandwellnesssummit.org/

Honigman, R.J., Phillips, K.A., \& Castle, D.J. (2004). A Review of Psychosocial Outcomes for Patients Seeking Cosmetic Surgery. Plastic and Reconstructive Surgery, 113(4), 12291237. 